\title{
Spectral analysis of auroral geomagnetic activity during various solar cycles between 1960 and 2014
}

\author{
Pieter Benjamin Kotzé \\ South African National Space Agency (SANSA), Space Science, Hermanus, South Africa \\ Correspondence to: Pieter Benjamin Kotzé (pkotze@ sansa.org.za)
}

Received: 25 May 2016 - Revised: 11 October 2016 - Accepted: 25 October 2016 - Published: 6 December 2016

\begin{abstract}
In this paper we use wavelets and Lomb-Scargle spectral analysis techniques to investigate the changing pattern of the different harmonics of the 27-day solar rotation period of the AE (auroral electrojet) index during various phases of different solar cycles between 1960 and 2014. Previous investigations have revealed that the solar minimum of cycles 23-24 exhibited strong 13.5- and 9.0-day recurrence in geomagnetic data in comparison to the usual dominant 27.0-day synodic solar rotation period. Daily mean AE indices are utilized to show how several harmonics of the 27day recurrent period change during every solar cycle subject to a $95 \%$ confidence rule by performing a wavelet analysis of each individual year's AE indices. Results show that particularly during the solar minimum of 23-24 during 2008 the 27-day period is no longer detectable above the $95 \%$ confidence level. During this interval geomagnetic activity is now dominated by the second (13.5-day) and third (9.0-day) harmonics. A Pearson correlation analysis between $\mathrm{AE}$ and various spherical harmonic coefficients describing the solar magnetic field during each Carrington rotation period confirms that the solar dynamo has been dominated by an unusual combination of sectorial harmonic structure during 23-24, which can be responsible for the observed anomalously low solar activity. These findings clearly show that, during the unusual low-activity interval of 2008, auroral geomagnetic activity was predominantly driven by high-speed solar wind streams originating from multiple low-latitude coronal holes distributed at regular solar longitude intervals.
\end{abstract}

Keywords. Magnetospheric physics (solar windmagnetosphere interactions)

\section{Introduction}

Geomagnetic activity is driven by solar activity which varies on different timescales, of which the $\sim 11$-year solar cycle is one of the best-known periodicities. This is because the Sun is a variable star that fluctuates and oscillates on various timescales ranging from seconds to several years. In particular solar activity rises and falls with the 11-year solar cycle, which has a direct influence on the near-Earth space environment, resulting in geomagnetic activity that also shows solar cycle dependence but is more complex than seen in sunspot numbers, radio flux, flares, and CMEs (coronal mass ejections; Hathaway, 2015). Lockwood et al. (1999) pointed out the presence of multi-cycle trends in geomagnetic activity that may be related to changes in solar magnetism; e.g. during solar maximum the Sun's magnetic field is predominantly toroidal, changing gradually during the declining phase to a poloidal structure (Solanki et al., 2006). In addition, a gradual transition from solar maximum dominated by coronal mass ejection activity to solar minimum activities dominated by regions of open flux coronal holes takes place during this phase of the solar cycle. The dipole solar magnetic field also varies between small values during solar maximum to maximum strength during solar minimum (Legrand and Simon, 1991). Therefore, when the geomagnetic field interacts with the solar wind, various amounts of energy are transferred to the Earth's magnetosphere depending on whether it is the solar maximum or solar minimum phase of a particular solar cycle. Geomagnetic variations can be observed at middle and low latitudes via the Dst index, as well as auroral regions where electrojet currents are dominant, as reflected in the behaviour of the AE (auroral electrojet) index. The auroral electrojet index is derived from variations measured in the horizontal component of the geomagnetic field recorded at 12 observatories located at high 


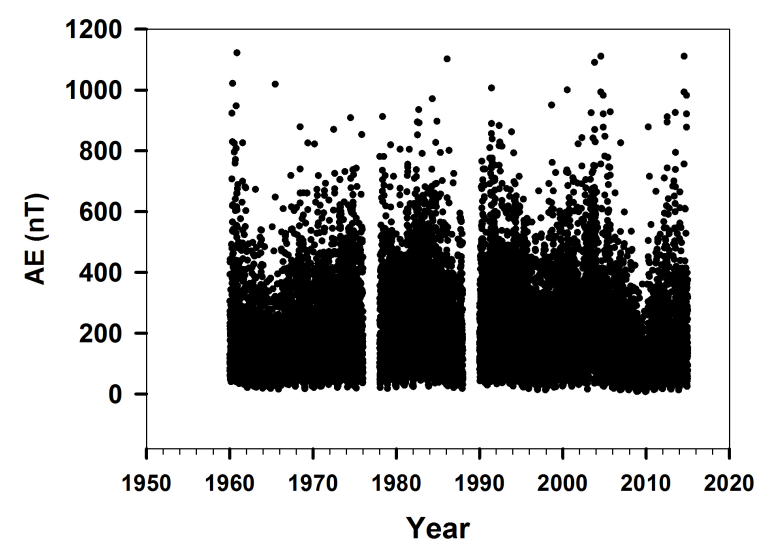

Figure 1. The AE time series from 1960 to 2013.

latitudes ranging between 61 and $70^{\circ}$ in the Northern Hemisphere. This index was first introduced by Davis and Sugiura (1966) to monitor magnetospheric substorms as well as the coupling between the interplanetary magnetic field and Earth's magnetosphere.

Files containing the $1 \mathrm{~h}$ AE indices for the period 19602014 were downloaded from the World Data Centre for Geomagnetism in Kyoto, Japan (http://wdc.kugi.kyoto-u.ac.jp/ aedir/index.html), which also included the daily mean values. These daily mean values were arranged and sorted according to each individual annual interval. The pattern of the long-term behaviour of the AE time series over several solar cycles between 1960 through to 2014 can be seen in Fig. 1, with no data available for 1976, 1977, 1988, and 1989.

A distinctive feature in Fig. 1 is the large and seemingly bursty excursions indicating auroral magnetic disturbances as well as very low levels of activity around 2010 . The main aim of this study is to investigate the evolution of the 27-day solar rotation period and its different harmonics in the $\mathrm{AE}$ time series on an annual basis.

All graphs and plots in this paper were produced using the SigmaPlot (www.systat.com) plotting package, while the contour plots of Figs. 2 and 3 were generated using the Interactive Data Language (IDL) (http://www.exelisvis. com/ProductsServices/IDL.aspx) wavelet applet. Two spectral analysis approaches are employed in this paper to perform a spectral analysis of auroral geomagnetic time series behaviour during various stages of several solar cycles between 1960 and 2014, namely the Lomb-Scargle (Lomb, 1976; Scargle, 1982) periodogram method and the Morlet (Morlet et al., 1982; Torrence and Compo, 1998) wavelet technique. Power spectra obtained from both methods were used to analyse the behaviour of different harmonics of the 27-day periodicity for all solar cycles since 1960.

\section{Spectral analysis and Pearson correlations}

Spectral analysis techniques such as wavelets and Fourier applications are well-established methods for revealing periodicities on various timescales in time series. Determining periodicities in incomplete or unevenly sampled time series for a given variable is not directly possible with methods such as fast Fourier transform (FFT) and may require some degree of interpolation to fill in gaps. An alternative is the Lomb-Scargle method (Lomb, 1976; Scargle, 1982), which estimates a frequency spectrum based on a least squares fit of unevenly sampled data to represent a signal as the sum of sine and cosine functions of infinite duration. The Fortran computer algorithm of Press et al. (1992, chapter 13.8) was applied to all daily mean observations for each individual year between 1960 and 2014 .

On the other hand wavelet analysis is useful to decompose a time series into time-frequency space and to establish the modes of variability, particularly when the time series under investigation contains non-stationary power at different periods. As the wavelet transform is a localized transform in both space (time) and frequency, this property can be applied to extract information from the signal, which is not possible with Fourier techniques (Daubechies, 1990). For the purposes of this investigation the Morlet wavelet (Morlet et al., 1982; Torrence and Compo, 1998), $\varphi(\tau)$ consisting of a plane wave modulated by a Gaussian distribution, was used:

$\varphi(\tau)=\pi^{-1 / 4} e^{i \omega \tau} e^{-\tau^{2} / 2}$,

where $\omega$ and $\tau$ are respectively the non-dimensional frequency and time parameters. The unique characteristic of wavelets to investigate both the periodicity content and localization in space and time is possible by representing signals $f(\tau)$ by series such as

$f(\tau)=\sum_{j=-\infty}^{\infty} \sum_{k=-\infty}^{\infty} c_{k}^{j} \varphi_{k}^{j}(\tau)$

where $\varphi_{k}^{j}=\varphi\left(2^{j} \tau-k\right)$ are defined as the mother (Morlet in this case) wavelets and $c_{k}^{j}$ as the wavelet coefficients whose amplitude (power) is characteristic of the signal they represent. Similar to our investigation of K-index behaviour (Kotzé, 2015), wavelets of order 5 were chosen as they have symmetric characteristics which make them suitable to represent high-order polynomials, necessary to identify periodicities in time series consisting of daily mean values.

Both above-mentioned methods were used to obtain the spectral contents of the AE index time series at each annual interval. The Lomb-Scargle method in particular served to verify the Morlet wavelet analysis results. Wavelet transforms were also applied by Balasis et al. (2006) to perform a fractal spectral analysis of the 2001 Disturbance stormtime (Dst) index time series at solar maximum, while Zaourar et al. (2013) performed a wavelet-based multiscale analysis 
of geomagnetic disturbances recorded at various magnetic observatories. Kunagu et al. (2013) also analysed 10 years of CHAMP (CHAllenging Minisatellite Payload) magnetic field data using wavelets and found external field periodicities of interest to global geomagnetic field models.

The correlation between the AE index and various spherical harmonic coefficients of the Wilcox magnetic field model, at 2.5 solar radii, were calculated using Pearson's technique. According to this method a linear correlation between two variables $(X$ and $Y$ ) can be determined via the Pearson correlation coefficient, which is the covariance of the two variables divided by the product of their standard deviations. Pearson correlation coefficients vary between +1 (strong correlation) through 0 (no correlation) to -1 (strong anti-correlation). The Fortran computer algorithm of Press et al. (1992, chapter 14.6) was employed to determine Pearson correlation coefficients.

\section{Results and discussion}

Morlet wavelet power spectra were obtained by analysing each individual year's daily mean AE indices from 1961 to 2013 in order to reveal the short-period geomagnetic activity behaviour during various phases of each solar cycle in this interval. This enabled the identification of second (13.5day), third (9.0-day), and fourth (6.7-day) harmonics of the fundamental 27-day solar rotation period and their individual behaviour during, particularly, solar maximum and solar minimum intervals at the $95 \%$ confidence level. A typical power spectrum for a solar maximum in 1991 is shown in Fig. 2. The dominance of the 27-day recurrence period can clearly be seen.

In contrast to a solar maximum interval, a solar minimum shows that the auroral geomagnetic activities are no longer dominated by the 27-day solar rotation period but that in fact the 13.5- and 9.0-day periodicities are playing a significant role. A typical example of a solar minimum AE wavelet power spectrum for 2008 is shown in Fig. 3.

Lomb-Scargle, being a variant of the Fourier spectral analysis methods, does not have the ability to analyse a time series into time-frequency space and to determine the dominant modes of variability, particularly when these time series contain non-stationary power located at different frequencies. This method was therefore employed to verify the wavelet results and to obtain the frequency (periodicity) content of the AE time series without localization in time. A typical example of Lomb-Scargle spectral analysis for a solar maximum year (2001) and a solar minimum year (2008) can be seen in Fig. 4.

What is also evident from this investigation is that the 27day solar rotation period is constantly present for all solar cycles between 1960 and 2014, but during the peculiar solar minimum period of cycles 23-24 the power spectrum is dominated by the second and third harmonics. The 9.0- and

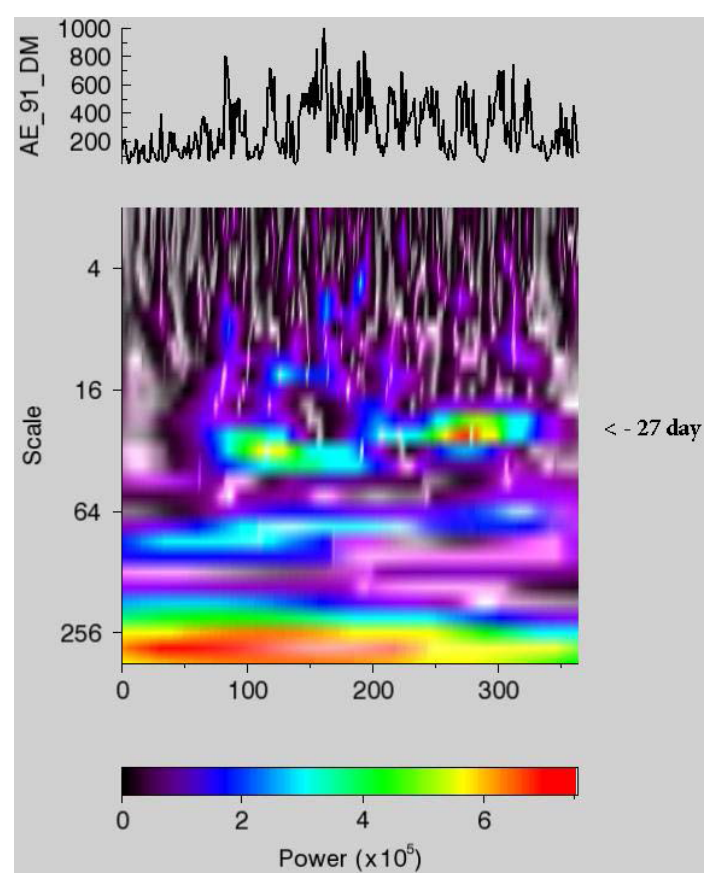

Figure 2. A Morlet AE power spectrum during solar maximum in 1991 showing the clear domination of the 27-day recurrence period. The top graph shows the daily mean AE indices used in the wavelet analysis for 1991 .

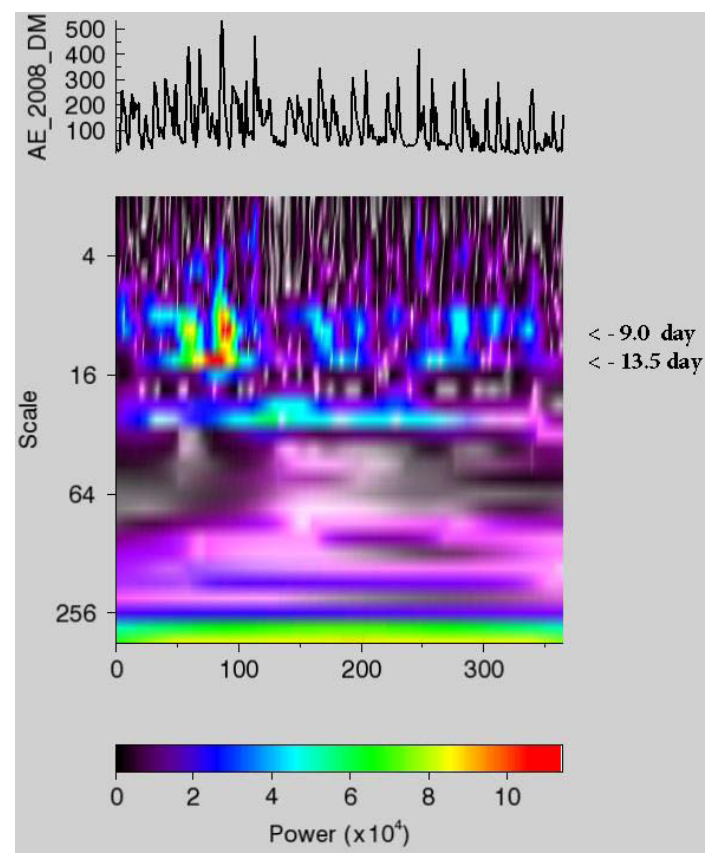

Figure 3. A Morlet wavelet power spectrum of the AE index during 2008 showing the dominant behaviour of the 13.5- and 9.0-day periodicities. The top plot shows the daily mean AE indices during 2008 used in the current wavelet analysis. 

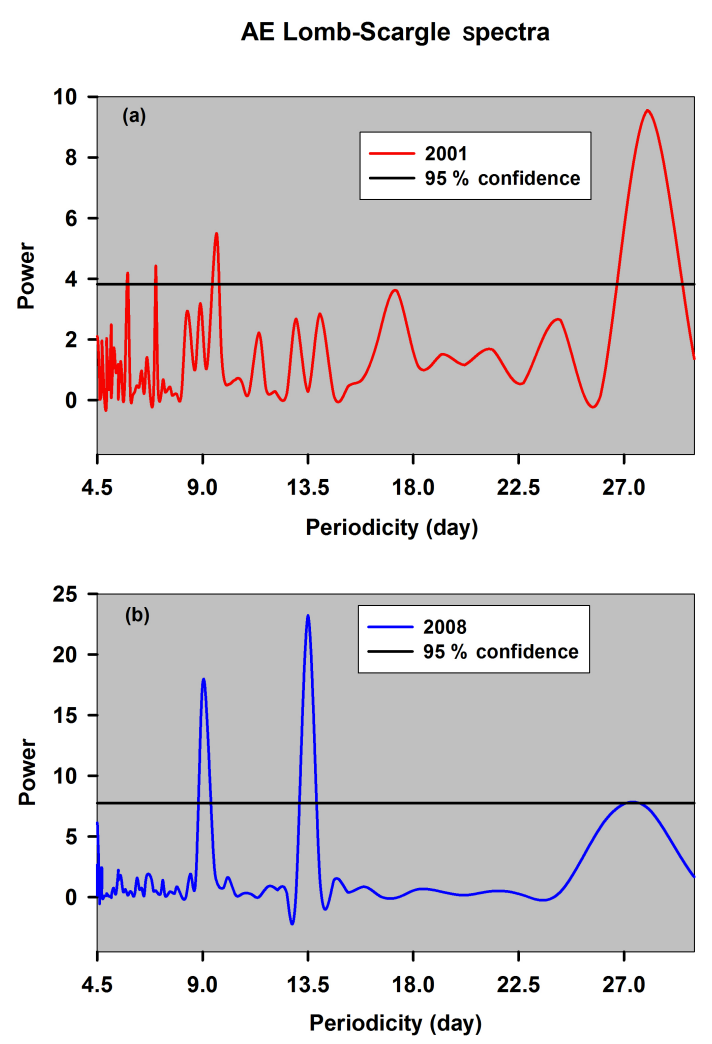

Figure 4. Lomb-Scargle spectral analysis of AE time series for 2001 (a) and 2008 (b) showing the dominance of the 27-day periodicity at solar maximum, while the 13.5- and 9.0-day periodicities are dominant during solar minimum at the $95 \%$ confidence level.

13.5-day periodicities seem to play an important and distinguishable role particularly during the downward phase and minimum periods of several solar cycles since 1960. This was quite evident during 1965, 1985, 1994, 1996, and 2008. A Lomb-Scargle analysis confirmed the wavelet analysis results. In particular for 2008, as shown in Fig. 4, only the 9.0and 13.5-day periods can be distinguished above the $95 \%$ level. Although a 26- and 28-day periodicity can be identified in the spectrum of the solar magnetic field during 2008, the solar activity is dominated by the 13.5 -day period. These figures and similar results obtained between 1960 and 2014 show that the different periodicities tend to appear without a fixed pattern during each individual year. In addition the average power also scales according to the particular harmonic. This phenomenon can most probably be ascribed to the dominance of CMEs during solar maximum geomagnetic storms, while fast streams originating in coronal holes are dominant during solar minimum periods. These results confirm previous findings regarding the behaviour of $\mathrm{K}$ indices at both high latitudes and midlatitudes (Kotzé, 2015). Several publications have also appeared in the scientific literature on results obtained by studying the behaviour and influence of solar cycle and solar wind parameters on geomagnetic activ-
Table 1. Pearson correlation coefficients between AE and dipole and quadrupole spherical harmonic coefficients of the solar magnetic field for 2008 .

\begin{tabular}{lr}
\hline $\begin{array}{l}\text { Spherical harmonic } \\
\text { coefficient }\end{array}$ & $\begin{array}{r}\text { Pearson } \\
\text { correlation }\end{array}$ \\
\hline$g_{1}^{0}$ & -0.11 \\
$g_{1}^{1}$ & -0.45 \\
$h_{1}^{1}$ & 0.82 \\
$g_{2}^{0}$ & 0.57 \\
$g_{2}^{1}$ & 0.19 \\
$g_{2}^{2}$ & 0.88 \\
$h_{2}^{1}$ & -0.88 \\
$h_{2}^{2}$ & -0.57 \\
Tilt angle & 0.76 \\
\hline
\end{tabular}

ity levels (e.g. Crooker et al., 1977; Kojima and Kakinuma, 1990; Mursula, 1999), as well as periodicities in the interplanetary magnetic field (Gonzalez and Gonzalez, 1987) and solar excursion phases during several solar cycles (Mursula and Zieger, 1998). Results obtained by these and other authors showed that the origin of periodicities in geomagnetic activities is closely linked to the domination of solar activity during solar cycle minimum by coronal holes (e.g. Sheeley Jr. et al., 1976), responsible for multi-component high-speed streams, while during solar maximum intervals CMEs are responsible for geomagnetic activity (Gosling et al., 1991).

Pearson linear correlation coefficients were determined between $\mathrm{AE}$ indices and all low-degree and low-order spherical harmonic coefficients as a function of Carrington rotation for every year since 1975 when these coefficients were starting to be determined by the Wilcox Solar Observatory. Table 1 shows results obtained for 2008 during the very low minimum of cycles 23-24.

From this table we can clearly see a strong correlation between $\mathrm{AE}$ and particularly the sectorial harmonic coefficients $h_{1}^{1}$ and $g_{2}^{2}$, while the dipole tilt angle of the solar magnetic field also exhibits an unusually strong correlation with $\mathrm{AE}$ during 2008.

\section{Conclusions}

Spectral analysis of the auroral geomagnetic activity as represented by the AE index has been performed for several solar cycles between 1960 and 2014 using techniques such as Morlet wavelet analysis and Lomb-Scargle. In particular it has been revealed that the 27-day solar rotation period dominates above the $95 \%$ confidence level during solar maximum years due primarily to the occurrence of CME-driven mag- 
netic storms. In contrast, solar minimum periods are characterized by the domination of 13.5- and 9.0-day periodicities above the $95 \%$ confidence level, which is a result of geomagnetic storms to a large extent being driven by coronalhole activities in these intervals. This is particularly evident during the extensive solar minimum period observed during cycles 23-24.

Lei et al. (2008) investigated CHAMP neutral densities for 2005 and revealed the presence of a 9-day periodicity in the data. A spectral analysis of the data showed strong evidence that the upper atmosphere is modulated by rotating coronal holes that have a dominant 27 -day periodicity as well (see, e.g., Fig. 2, Lei et al., 2008) above the $95 \%$ significance level. Similar behaviour was also observed by Thayer et al. (2008) when investigating thermospheric density oscillations resulting from high-speed solar wind streams during 2006, reporting periodic oscillations at 4-5-, 6-7-, and 9-11day periods, with the 7-day period the dominating oscillation. It is also quite clear (see, e.g., Fig. 2, Thayer et al., 2008) that the 27-day period is still present well above the $95 \%$ significance level. A separate investigation by Love et al. (2012) using autocorrelation techniques, clearly identified 6.7- and 9.0-day geomagnetic recurrence intervals in the $a a$ time series during the unusual 23-24 solar cycle minimum, in addition to the presence of a dominating 27-day periodicity (see, e.g., Fig. 1, Love et al., 2012).

Results obtained in this investigation using both wavelet and Lomb-Scargle spectral analysis methods to investigate the behaviour of auroral geomagnetic activity during cycles 23-24 support the findings of all three above-mentioned investigations during 2005 and 2006. In both 2005 and 2006 the AE index, which is a proxy for ionospheric convection behaviour, is characterized by the appearance of 6.7-, 9.0-, and 13.5-day periodicities, while the 27-day synodic oscillation is still the dominating periodicity above the $95 \%$ significance level. However, during 2008 which is characterized by the least number of sunspots since 1913, the spectrum of the auroral geomagnetic activity shows dominant 9.0- and 13.5day periodicities well above the $95 \%$ confidence level, while the power of the 27-day periodicity is significantly reduced in comparison to these prominent higher harmonics. It is worth mentioning that the power of 27-day period is now below the $95 \%$ significance level during 2008 as can be seen in Fig. 4 . This new finding shows that during the unusual low-activity interval of 2008 that auroral geomagnetic activity was predominantly driven by high-speed solar wind streams originating from multiple low-latitude coronal holes distributed at regular solar longitude intervals.

A study by Richardson et al. (2001) concluded that storms associated with high-speed solar wind streams originating in coronal holes are most prevalent during the decay and minimum phase of the solar cycle which are generally small or medium in size in comparison to solar maximum conditions. The declining phase of cycle 23 and the following unusually low and extended minimum of cycles $23-24$ were different to previous solar cycles (e.g. Russell et al., 2010). A previous investigation of $\mathrm{K}$ indices (Kotzé, 2015) also revealed that the solar magnetic field was dominated by the 13.5-day period during the solar minimum of cycles $23-24$. This and the findings in this paper show that the peculiar behaviour of geomagnetic activity in the auroral region can most probably be attributed to the non-axisymmetric heliosphere that drives the observed recurrences. Since the Sun controls the heliosphere, we can safely conclude that the solar dynamo was in a state of unusual asymmetry during that time. The strong Pearson correlations we obtained between auroral activity as measured by the $\mathrm{AE}$ index and particularly the low-degree sectorial spherical harmonics is therefore an indication of the non-axisymmetric structure in the solar poloidal magnetic field having a profound influence on the heliosphere. The indications are strongly in favour of the sectorial structure in the solar magnetic field being responsible for the dominant 13.5- and 9.0-day auroral geomagnetic activity seen during minimum 23-24, supporting the investigation by Love et al. (2012) using $a a$ indices. This investigation therefore provides strong support that prominent recurrence intervals detected in auroral geomagnetic activity (AE indices) can be linked to an asymmetric solar magnetic field that existed during the peculiar minimum of solar cycles 23-24 (see Fig. 1 in Kotzé, 2015). This manuscript can be regarded as a followup to our previous publication on short-period K-index behaviour at both mid- and high latitudes (Kotzé, 2015).

\section{Data availability}

Files containing the $1 \mathrm{~h} \mathrm{AE}$ indices for the period 19602014 were downloaded from the World Data Centre for Geomagnetism in Kyoto, Japan (http://wdc.kugi.kyoto-u.ac.jp/ aedir/index.html), which also included the daily mean values (World Data Center C2 for Geomagnetism, 2016).

Acknowledgements. The efforts in running the auroral magnetic observatories to measure and archive data for the derivation of $\mathrm{AE}$ indices are greatly appreciated.

The topical editor, G. Balasis, thanks the two anonymous referees for help in evaluating this paper.

\section{References}

Balasis, G., Daglis, I. A., Kapiris, P., Mandea, M., Vassiliadis, D., and Eftaxias, K.: From pre-storm activity to magnetic storms: a transition described in terms of fractal dynamics, Ann. Geophys., 24, 3557-3567, doi:10.5194/angeo-24-3557-2006, 2006.

Crooker, N. U., Feynman, J., and Gosling, J. T.: On the high correlation between long-term averages of solar wind speed and geomagnetic activity, J. Geophys. Res., 82, 1933-1937, 1977.

Daubechies, I.: The wavelet transform, time-frequency localization and signal analysis, IEEE T. Inform. Theory, 36, 961-1005, 1990. 
Davis, T. N. and Sigiura, M.: Auroral electrojet activity index AE and its universal time variations, J. Geophys. Res., 71, 785-801, 1966.

Gonzalez, A. L. C. and Gonzalez, W. D.: Periodicities in the interplanetary magnetic field polarity, J. Geophys. Res., 92, 43574375, 1987.

Gosling, J. T., McComas, D. J., Phillips, J. L., and Bame, S. J.: Geomagnetic activity associated with Earth passage of interplanetary shock disturbances and coronal mass ejections, J. Geophys. Res., 96, 7831-7839, 1991.

Hathaway, D. H.: The solar cycle, Living Rev. Solar Phys., 12, doi:10.1007/lrsp-2015-4, 2015.

Kojima, M. and Kakinuma, T.: Solar cycle dependence of global distribution of solar wind speed, Space Sci. Rev., 53, 173-222, 1990.

Kotzé, P. B.: Spectrum analysis of short-period K index behaviour at high and mid-latitudes, Ann. Geophys., 33, 31-37, doi:10.5194/angeo-33-31-2015, 2015.

Kunagu, P., Balasis, G., Lesur, V., Chandrasekhar, E., and Papadimitriou, C.: Wavelet characterization of external magnetic sources as observed by CHAMP satellite: evidence for unmodeled signals in geomagnetic field models, Geophys. J. Int., 192, 946-950, doi:10.1093/gji/ggs093, 2013.

Legrand, J. P. and Simon, P. A.: A two component solar cycle, Sol. Phys., 131, 187-209, 1991.

Lei, J., Thayer, J. P., Forbes, J. M., Sutton, E. K., and Nerem, R. S.: Rotating solar coronal holes and periodic modulation of the upper atmosphere, Geophys. Res. Lett., 35, L10109, doi:10.1029/2008GL033875, 2008.

Lockwood, M., Stamper, R., and Wild, M. N.: A doubling of the Sun's coronal magnetic field during the past 100 years, Nature, 399, 437-439, 1999.

Lomb, N. R.: Least-squares frequency analysis of unequally spaced data, Astrophys. Spa. Sci., 39, 447-462, doi:10.1007/BF00648343, 1976.

Love, J. J., Rigler, E. J., and Gibson, S. E.: Geomagnetic detection of the sectoral solar magnetic field and the historical peculiarity of minimum 23-24, Geophys. Res. Lett., 39, L04102, doi:10.1029/2011GL050702, 2012.
Morlet, J., Arens, G., Forgeau, I., and Giard, D.: Wave propagation and sampling theory, Geophys., 47, 203-236, 1982.

Mursula, K.: Simultaneous occurrence of mid-term periodicities in solar wind speed, geomagnetic activity and cosmic rays, in: UPAP Proceedings of the 26th International Cosmic Ray Conference 7, edited by: Kieda, D., Salamon, M., and Dingus, B., 123-126, 17-25 August 1999.

Mursula, K. and Zieger, B.: Solar excursion phases during the last 14 solar cycles, Geophys. Res. Lett., 25, 1851-1854, 1998.

Press, W. H., Teukolsky, S. A., Vetterling, W. T., and Flannery, B. P.: Numerical Recipes in Fortran: The Art of Scientific Computing, Cambridge Univ. Press, UK, 963 pp., 1992.

Richardson, I. G., Cane, H. V., and Cliver, E. W.: Sources of geomagnetic storms for solar minimum and maximum conditions during 1972-2000, Geophys. Res. Lett., 28, 2569-2572, 2001.

Russell, C. T., Luhmann, J. G., and Jian, L. K.: How unprecedented a solar minimum?, Rev. Geophys., 48, RG2004, doi:10.1029/2009RG000316, 2010.

Scargle, J. D.: Studies in astronomical time series II, Statistical aspects of spectral analysisof unevenly spaced data, Astrophys. J., 263, 835-853, doi:10.1086/160554, 1982.

Sheeley Jr., N. R., Harvey, J. W., and Feldman, W. C.: Coronal holes, solar wind streams, and recurrent geomagnetic disturbances 1973-1976, Sol. Phys., 49, 271-278, 1976.

Solanki, S. K., Inhester, B., and Schüssler, M.: The solar magnetic field, Rep. Prog. Phys., 69, 563-668, 2006.

Thayer, J. P., Lei, J., Forbes, J. M., Sutton, E. K., and Nerem, R. S.: Thermospheric density oscillations due to periodic solar wind high-speed streams, J. Geophys. Res., 113, A06307, doi:10.1029/2008JA013190, 2008.

Torrence, C. and Compo, G. P.: A practical guide to wavelet analysis, B. Am. Meteorol. Soc., 79, 61-78, 1998.

World Data Center C2 for Geomagnetism: Geomagnetic Auroral Electrojet Index, Kyoto Graduate School of Science, Kyoto University, Kyoto, Japan, available at: http://wdc.kugi.kyoto-u.ac.jp/ aedir/index.html, 2016.

Zaourar, M., Hamoudi, M., Mandea, M., Balasis, G., and Holschneider, M.: Wavelet-based multiscale analysis of geomagnetic disturbance, Earth Planets Space, 65, 1525-1540, 2013. 